\title{
IMPLANTACIÓN DE UNA APLICACIÓN MÓVIL PARA PPGIS DESTINADA A DESLIZAMIENTOS E INTEGRADA A UNA IDE
}

\author{
IMPLEMENTATION OF A MOBILE APPLICATION FOR LANDSLIDES ORIENTED \\ TO PPGIS AND INTEGRATED TO A SDI
}

\author{
${ }^{1}$ ROSARIO ACHIG, ${ }^{1}$ VILLIE MOROCHO, ${ }^{1}$ FABIÁN SANTANDER, ${ }^{2}$ NATALIA PACURUCU \\ ${ }^{1}$ DEPARTAMENTO DE CIENCIAS DE LA COMPUTACIÓN DE LA UNIVERSIDAD DE CUENCA. \\ ${ }^{2}$ FACULTAD DE ARQUITECTURA Y URBANISMO DE LA UNIVERSIDAD DE CUENCA. \\ Av. 12 de Abril y Agustín Cueva. Cuenca, Ecuador. e-mail: rosario.achig@ucuenca.edu.ec, villie. \\ morocho@ucuenca.edu.ec, fabian.santander@ucuenca.edu.ec, natalia.pacurucu@ucuenca.edu.ec.
}

Recibido: 15 de agosto 2018 /Aceptado: 25 de septiembre 2018

\section{RESUMEN}

Se ha generado una aplicación móvil para participación ciudadana aplicada a Sistemas de Información Geográfica -SIG- para que la población pueda proporcionar datos específicos sobre zonas que hayan sufrido deslizamientos o propensas a este fenómeno, de manera que los investigadores o tomadores de decisiones puedan contar con datos actualizados y geo localizados de estas zonas. En base a información proporcionada por la Secretaría de Gestión de Riesgos -SGR- y la experiencia del personal del proyecto, se definieron los perfiles y contenidos de la encuesta implementada a través de la aplicación móvil. Posteriormente se utilizó esta aplicación para levantamiento de información en determinadas áreas de la ciudad de Cuenca en las que se realizaron estudios sobre deslizamiento. Los resultados de la información levantada pueden ser visualizados en la plataforma de Infraestructura de Datos Espaciales de la Universidad de Cuenca -IDE UCuenca-, en diferentes formatos. Adicionalmente, el contenido de la aplicación móvil fue validado por el personal de la SGR.

Palabras clave: Deslizamientos, Participación Ciudadana, PP GIS, Aplicación Móvil, IDE.

\begin{abstract}
A mobile application for citizen participation applied to Geographic Information Systems -GIS- has been created so that population can provide specific data on areas with landslides or susceptible to this phenomenon. With this information, researchers or decision makers can have updated and geo-located data from these areas. Based on information provided by the Secretariat of Risk Management -SGR- and the experience of the project staff, the profiles and contents of the survey implemented through the mobile application were defined. Later this application was used to get information in certain areas of the city of Cuenca in which studies of landslides were performed. The results of the information obtained can be visualized in the Spatial Data Infrastructure platform of the University of Cuenca-Ucuenca SDI-, in different formats. Additionally, the content of the mobile application was validated by the staff of the SGR.
\end{abstract}

Keywords: Landslides, Public Participation, PP GIS, Mobile Application, IDE. 


\section{INTRODUCCIÓN}

La participación ciudadana tiene un impacto directo en la formulación, aplicación de políticas y la gestión del territorio. Los ciudadanos son vistos como una parte integral del proceso de gobernanza y su participación activa, se considera esencial para las decisiones críticas que enfrenta una comunidad (Callahan 2007).

Por otro lado, las Tecnologías de la Información y la Comunicación (TIC), en la Sociedad de la Información (SI), no solamente surgen como herramientas para ser aplicadas, sino como procesos para ser desarrollados (Castells 2008), lo que permite que los usuarios puedan tomar el control de las tecnologías. Así, las TIC deben estar al servicio de la comunidad, permitiendo el acceso a la información y concientizando a la población sobre ello. Las NTIC (Nuevas TIC) han generado un nuevo espacio de democracia para que la ciudadanía pueda ejercer su derecho a involucrarse en las decisiones del Estado. Una de las herramientas de las NTIC que promueve la participación pública o participación ciudadana es la Public Participation Geographic Information Systems (PP GIS). Dicho término es usado para referirse a un campo de los sistemas de información geográfica (SIG), que busca mejorar la participación del ciudadano y fomentar el empoderamiento de las organizaciones no gubernamentales, los grupos de base y comunidades locales (Sheppard et al. 1999; Sieber 2006).

En (Pearce 2003) se describe la importancia de la integración de la planificación comunitaria a la planificación para la gestión de desastres. Se detalla la visión de un enfoque exitoso en la mitigación sostenible de riesgos a través de la participación derivada de la vinculación con el nivel local en la toma de decisiones.

Una investigación de NTIC aplicada a participación ciudadana (Abhik y Toshniwal 2013) permite utilizar datos de medios sociales georreferenciados para facilitar la búsqueda y ubicación de sub-eventos con la finalidad de facilitar las actividades de ayuda y rescate durante los eventos por desastres naturales.

Para el caso de Ecuador, la promoción de la participación ciudadana ha tomado auge, sin embargo, estas prácticas aún no han sido instauradas. Es así que la tecnología no se ha aprovechado para estos fines y se necesita de un primer acercamiento que pueda incorporar el conocimiento local en la ordenación territorial (Pacurucu y Morocho 2017).

La iniciativa que se presenta a continuación, ha explorado nuevas formas de involucrar a la población a través de la recopilación de información cartográfica relevante que pueda convertirse en insumos para la planificación territorial, de modo que la población no solamente proporcione datos, sino también se capacite y concientice sobre cómo usar el suelo y con ello evitar los asentamientos en zonas vulnerables (Morocho 2013). 
Este proceso se realiza a través de una aplicación móvil orientada a deslizamientos, la cual fue probada con un grupo de estudiantes simulando el uso de un ciudadano común y que permitió levantar tres zonas de estudio. Finalmente, se validó la encuesta por parte de personal técnico de la SGR para tener la versión final que estará puesta a disposición del ciudadano por parte de la SGR. La información levantada es almacenada en línea para su posterior análisis y visualización en la plataforma IDEUCuenca (Morocho y Santander 2015).

\section{DISEÑO DE LA APLICACIÓN MÓVIL}

El principal objetivo de la aplicación móvil es reportar la información de los eventos registrados al servidor de la plataforma IDE-UCuenca, la cual consta de dos partes, una fotografía geo-referenciada y una encuesta relaciona al mismo. Es necesario que las interfaces sean lo más claras y sencillas posibles, evitando unir en un mismo formulario un gran número y variedad de parámetros (preguntas). Para ello se ha diseñado una encuesta dinámica, en la cual las preguntas van a ir surgiendo de acuerdo a las opciones que el usuario vaya seleccionando (encuesta tipo árbol), como se puede ver en la Figura 1. De esta manera el usuario visualizará únicamente los parámetros necesarios, los cuales estarán organizados en varios formularios, mostrando un ambiente intuitivo que facilita y optimiza el tiempo en el envío de la información.

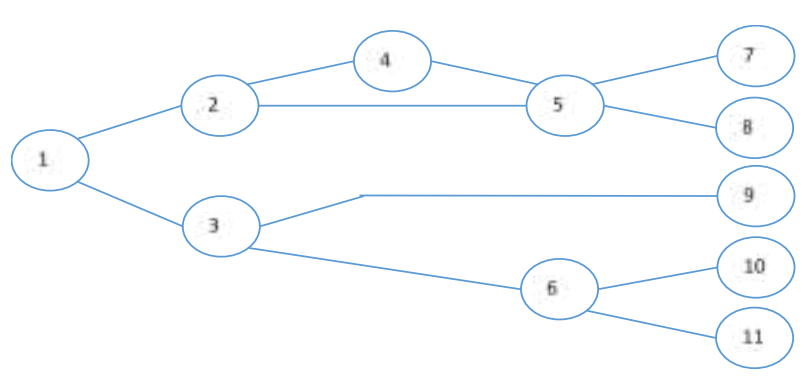

Figura 1. Encuesta tipo árbol

El contenido de la encuesta, se realizó tomando como base una ficha de campo para estimación de vulnerabilidades, elaborada por la SGR, e incorporando la experiencia del personal del proyecto en el área de deslizamientos.

La ficha de campo de la SGR contiene información general para estimación de varios tipos de vulnerabilidad. Se divide en 5 secciones: ubicación, registro de la observación, exposición, fragilidad y resiliencia. En este análisis, se han considerado las secciones y parámetros significativos para cumplir el objetivo de integrar a la plataforma IDEUCuenca, la información obtenida a través de la participación ciudadana.

En lo referente a los usuarios de la aplicación, se han definido dos perfiles: uno para el ciudadano en general que desea reportar un evento y otro para un usuario especializado o 
técnico, que levantará información más detallada, y podría ser utilizado por funcionarios o voluntarios de la SGR.

\section{PERFILES DE LA APLICACIÓN MÓVIL \\ Perfil Ciudadano}

Este perfil tendrá secciones que podrán ser llenados de manera intuitiva por parte de la población de modo que la información esté disponible de manera inmediata por parte de la SGR para que pueda tomar las medidas pertinentes.

Ubicación: En esta sección se obtendrán automáticamente las coordenadas de localización a partir de la ubicación del dispositivo móvil. Adicionalmente, si el usuario lo desea puede tomar una fotografía del evento.

Registro de la observación: Se elige el tipo de amenaza, en este caso se presenta la opción de "Deslizamientos", sin embargo este proceso puede ser aplicado a otro tipo de evento como inundación, sismo, erupción volcánica, entre otros.

Exposición y fragilidad: En primer lugar el usuario podrá elegir si el evento que desea reportar afecta a una infraestructura o terreno.

En el caso de infraestructura deberá elegir el tipo: edificación (vivienda, hospital, etc.) o vía/puente. Para cualquiera de las opciones, deberá indicarse los años de construcción. En caso de edificación deberá indicarse el número de pisos, y en el caso de vía/puente deberá indicarse el tipo de vía y el estado de la red vial.

A continuación se solicitará información relacionada al deslizamiento, como la aparición de grietas, tiempo, ubicación, posibles causas, entre otras.

\section{Perfil Técnico}

Este perfil permitirá obtener información más especializada en la sección "Exposición y fragilidad"

En el caso de infraestructura deberá elegir el tipo: edificación (vivienda, hospital, etc.) o vía/puente. Para cualquiera de las opciones, deberá indicarse los años de construcción y la captación de agua potable.

En caso edificación deberá indicarse el número de pisos, sistema estructural, tipo de material de las paredes, tipo de material de la cubierta, sistema de alcantarillado, estado del sistema de alcantarillado. En el caso de vía/puente deberá indicarse el tipo de vía y el estado de la red vial.

De igual manera en la información relacionada al deslizamiento se solicitará más detalle. 


\section{DISEÑO DE BASE DE DATOS}

Para el almacenamiento de la información se contará con dos bases de datos: una relacional PostgreSQL, que guardará información vinculada al evento, y una base de datos espacial PostGIS que almacenará la información geográfica del mismo. En la Figura 2 se puede ver la relación que existe entre las dos bases de datos, relacionadas a través de un campo de identificación.

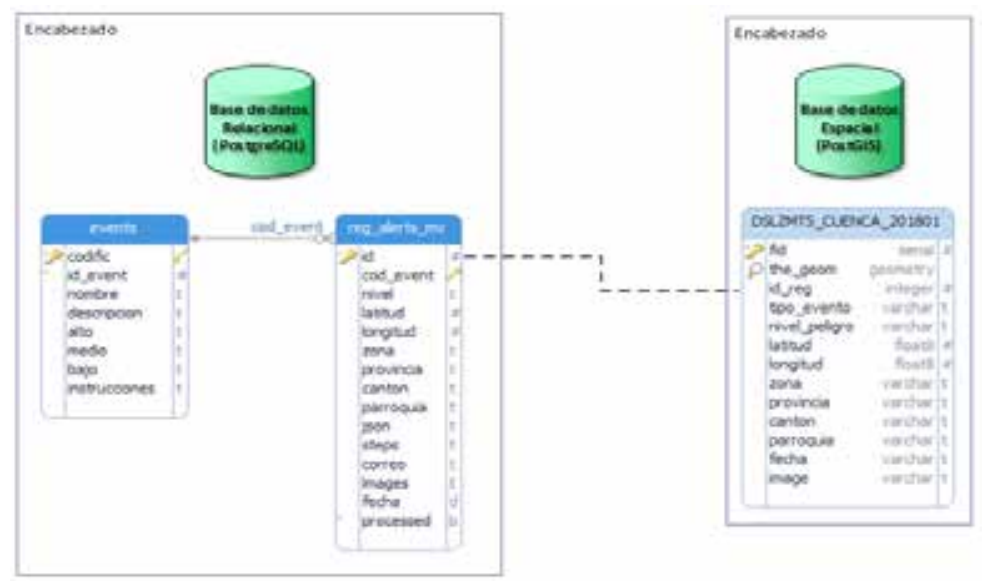

Figura 2. Diseño de bases de datos

\section{DESARROLLO DE LA APLICACIÓN MÓVIL}

Para desarrollar la aplicación móvil se consideró la funcionalidad de la encuesta tipo árbol. Adicionalmente, se codificó un sistema que permite interpretar un archivo JSON, el mismo que contiene todos los parámetros de la encuesta. Estos parámetros serán convertidos en formularios con diferentes elementos HTML (Cajas de texto, Cheklist, Labels, etc.), lo que permitirá actualizar las encuestas en la aplicación móvil de una manera dinámica. Como se puede ver en la Figura 3, el archivo JSON está almacenado en el servidor IDE UCuenca y las actualizaciones de los formularios se realizarán en el servidor, por lo tanto, si se realiza un cambio en el archivo JSON, no será necesario actualizar la aplicación.

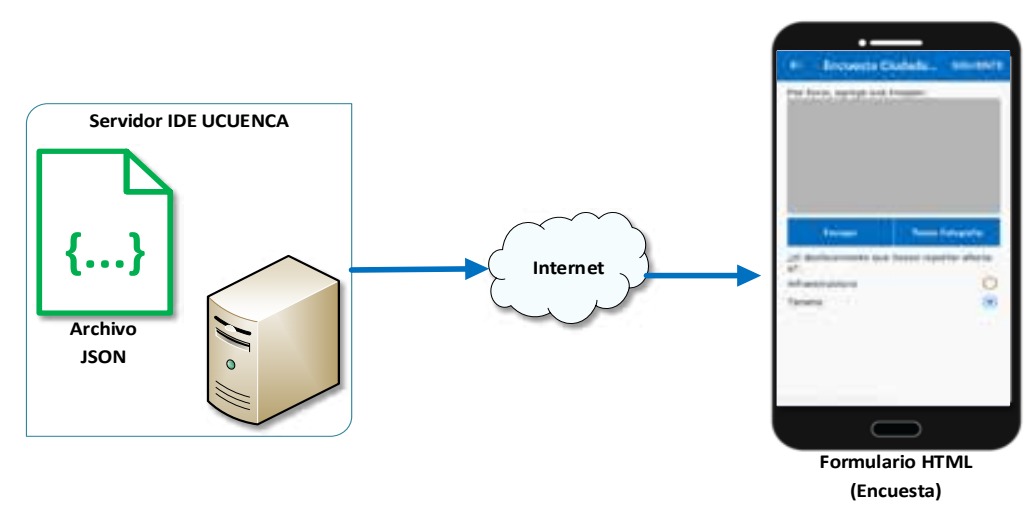

Figura 3. Obtención del archivo JSON en la Aplicación Móvil 
En la Figura 4 se expone un fragmento del archivo correspondiente a la encuesta que se va a realizar a un ciudadano, en formato JSON.

El archivo JSON puede ser modificado fácilmente, esto evita que la aplicación móvil sea rígida y modificar las encuestas directamente desde el código fuente.

Una vez finalizada la construcción del archivo JSON, la aplicación móvil visualizará de manera automática los formularios HTML que fácilmente pueden ser interpretados por el usuario, como se puede ver en la Figura 5.

Actualmente la generación del archivo JSON se está realizando de forma manual, pero en un futuro se pretende generar este archivo de forma dinámica mediante un entorno de administración gráfica.

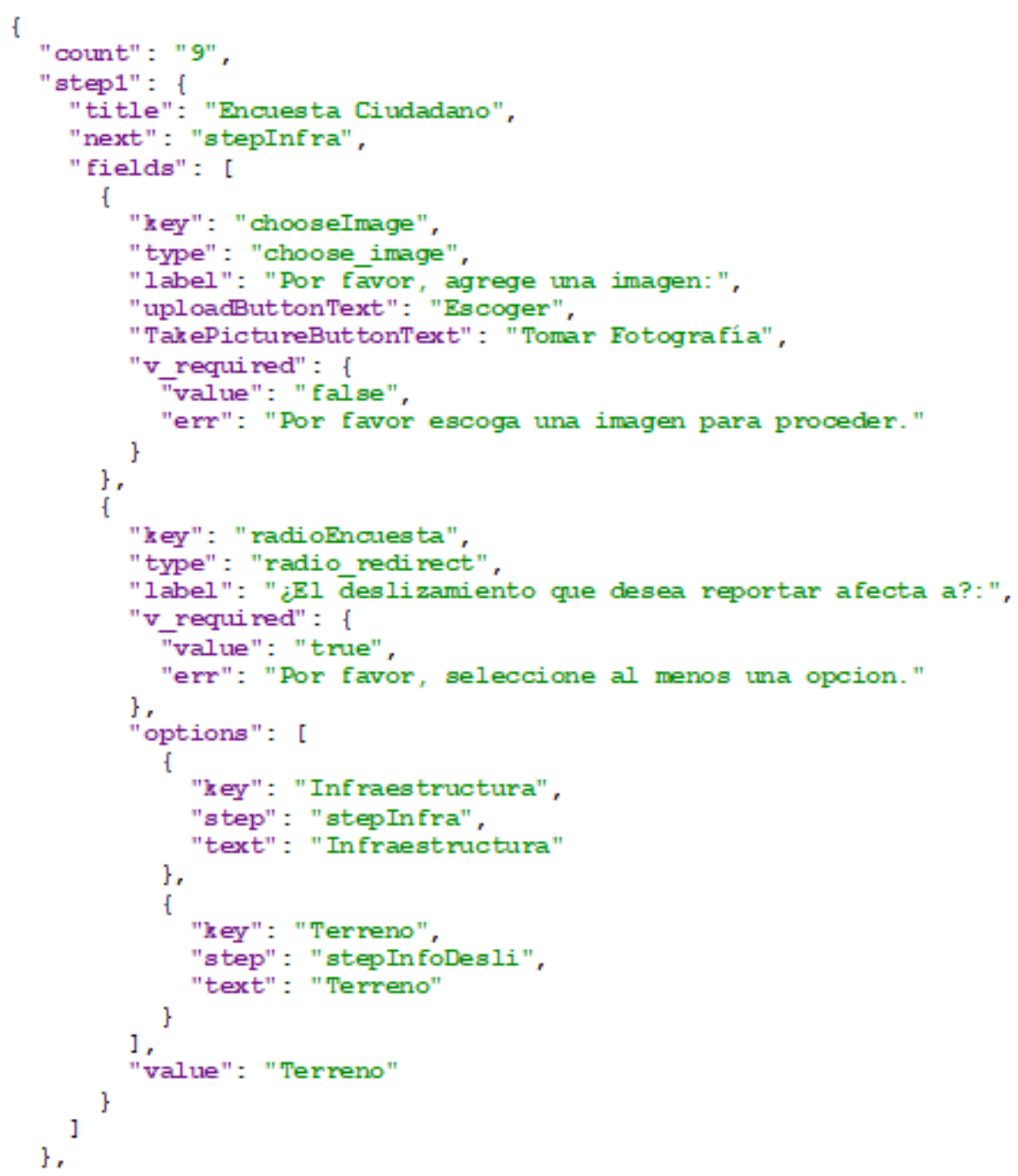

Figura 4. Extracto de archivo JSON 

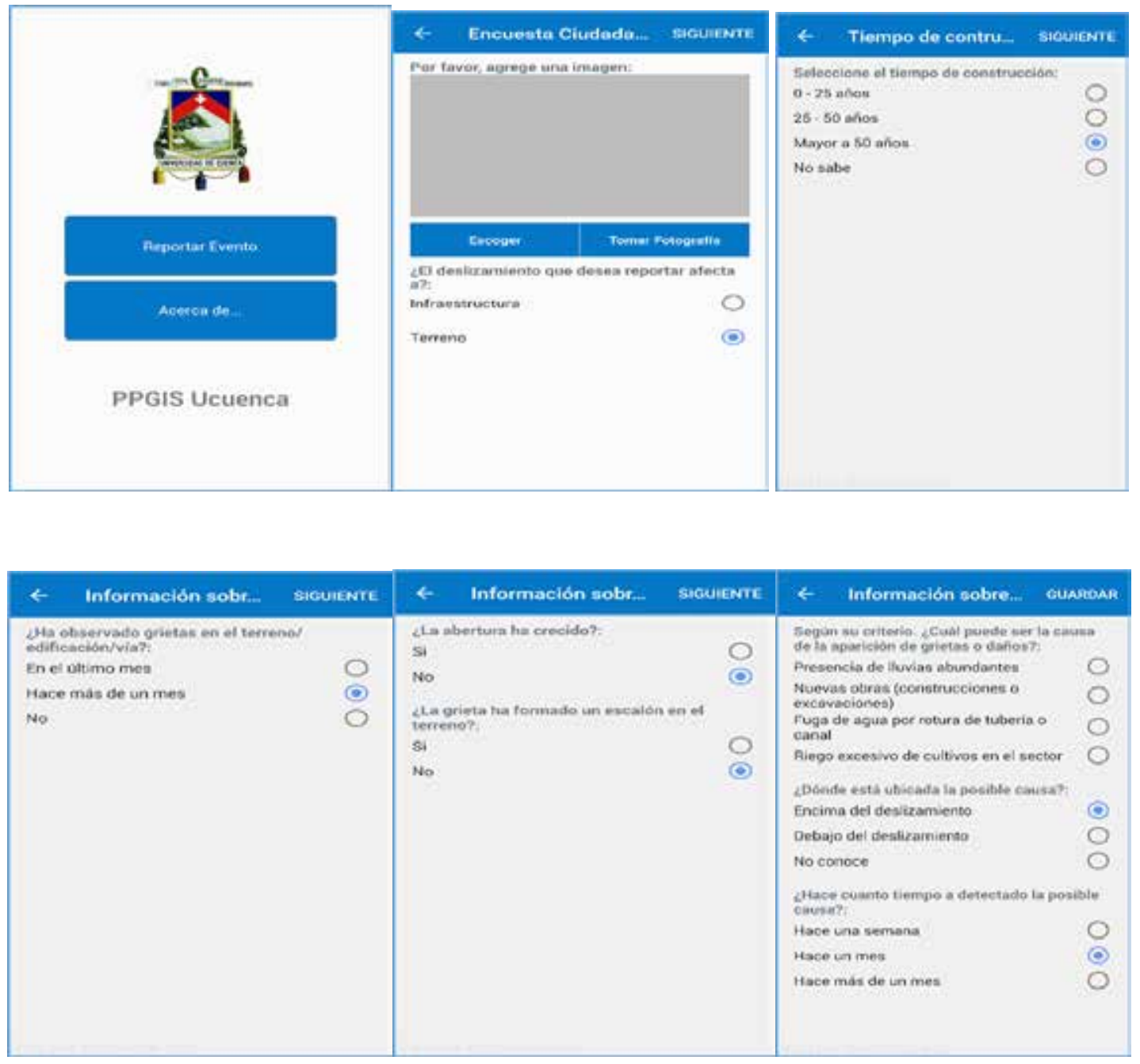

Figura 5. Capturas de formularios de la Encuesta

\section{INTEGRACIÓN DE LA APLICACIÓN MÓVIL CON LA IDE-UCUENCA}

En la Figura 6 se muestra un esquema general del proceso secuencial y automatizado que permitirá almacenar la información en la base de datos espacial y visualizarla en la plataforma IDE-UCuenca. Este proceso se desencadena cuando el usuario termina de reportar la información en su aplicación móvil.

A continuación se detalla el proceso completo:

a. Se guardan los eventos reportados en una tabla relacional de la Base de Datos PostgreSQL (para tener un registro del número de reportes realizados), como se puede ver en la Tabla 1. 


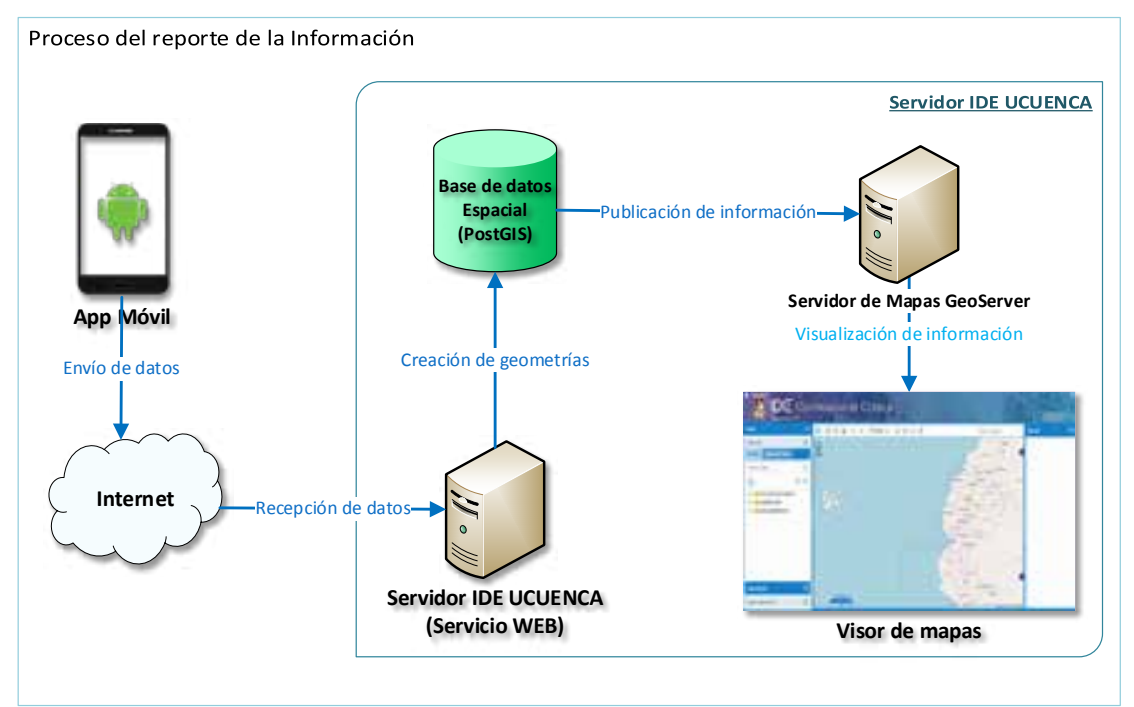

Figura 6. Proceso de envío de la información

Tabla 1. Registros de todos los eventos reportados mediante la Aplicación Móvil

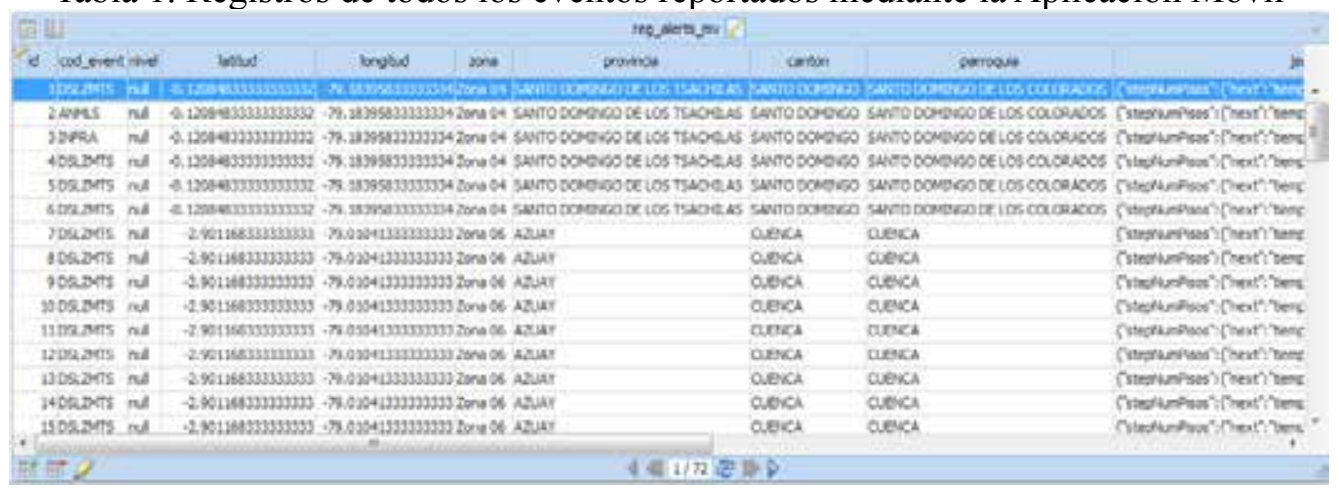

b. De acuerdo a las coordenadas reportadas por el dispositivo móvil: se calculan los siguientes datos: zona, provincia, cantón, parroquia.

c. Se generan geometrías (puntos georreferenciados) a partir de las coordenadas reportadas por la Aplicación Móvil y se almacenan en la base de datos espacial PostGIS.

d. Se clasifica la información receptada, que consiste en generar una tabla (capa) por mes mediante la siguiente información.

- Generación del nombre de las capas según el tipo de evento, cantón, año y mes.

- Generación de las tablas con sus respectivos nombres en la base de datos espacial. Si existe un reporte con un mismo tipo de evento en el mismo cantón y el mismo mes, se actualiza la tabla agregando nuevos puntos, caso contrario se genera una nueva tabla en la base de datos espacial, como se puede ver en la Tabla 2. 
Tabla 2. Base de Datos Espacial (Capa)

\begin{tabular}{|c|c|c|c|c|c|c|c|c|}
\hline \multicolumn{8}{|c|}{ 䀧再 } & \\
\hline Sod & the geom & idfseg & tipo_evento & melselgro & lastud & longtud & zona & \\
\hline & 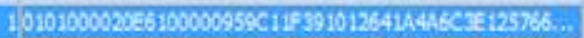 & 483 & SEST2AMIETOS & NED:0 & 2,20010383393 & \multicolumn{3}{|c|}{79,0109252 rara 061 . } \\
\hline & 2010100002066100000959 C $11 F 391012641$ A 4AGC $3 X 125766 \ldots$ & 440 & Desizanientos & MEOLO & $-2,9010363333$ & \multicolumn{3}{|c|}{$\cdot 79,010925$ Zans 06} \\
\hline & $30101000020 E 6100000959 \mathrm{C} 11 \mathrm{F39} 1012641 \mathrm{~A} 4 \mathrm{~A} 6 \mathrm{C} 3 \mathrm{E}$ 125766... & 450 & DESLZZMMENTOS & MED:O & $-2,9010383333$ & \multicolumn{3}{|c|}{$\cdot 79,010925$ Zona OS } \\
\hline & $40101000020 E 6100000959 \mathrm{C} 1 \mathrm{FF} 391012641 \mathrm{~A} 4 \mathrm{~A} G \mathrm{CC} 3 \mathrm{C} 125766 \ldots$ & 460 & DESUIZAMIENTOS & Meoto & $-2,9010383333$ & \multicolumn{3}{|c|}{$.79,010925$ zona 06} \\
\hline & $50101000020 E 6100000959 C 11 F 391012641$ A4ABC $3 E 125766 \ldots$ & 470 & SesLizaMientos & MEDIO & $-2,9010383333$ & \multicolumn{3}{|c|}{$.79,010925$ Zona 06 } \\
\hline & $60101000020 E 6100000959$ C11F391012641A4AGCXE1257766... & 480 & XEsIzantentos & MEDLO & $-2,9010383333$ & \multicolumn{3}{|c|}{$\cdot 79,010925$ zons 06} \\
\hline & $70101000020 E 6100000959 C 11 F 391012641$ A $4 A 6 C 3 E 125766 \ldots$ & 490 & DESLIZAMTENTOS & MED:O & $-2,9010383333$ & \multicolumn{3}{|c|}{$-79,010925$ Zana 06} \\
\hline & $80101000020 E 6100000$ 1F $68349 C F 0052641$ C 40 CF $15632766 \ldots .$. & 500 & EEs.zaMIENTOS & MEDto & $-2,9001283333$ & \multicolumn{3}{|c|}{$.79,0058966667$ zona 06} \\
\hline & $90101000020 E 6100000$ 1F6Bs 4SCF0052641C4DCF $15632766 \ldots$ & 510 & Desizamientos & MEDTO & $-2,9001283333$ & \multicolumn{3}{|c|}{$-79,0058966667$ Zona 06} \\
\hline & 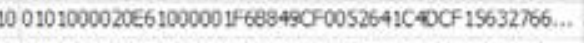 & 520 & DESLIZAMIENTOS & MEDto & $-2,9001283333$ & \multicolumn{3}{|c|}{79,0059966667 Zona 06} \\
\hline & $110101000020 E 6100000$ 1F68349CF0052641C4DCF $15632766 \ldots$ & 530 & DesulZaMiENTOS & MEOTO & $-2,9001283333$ & \multicolumn{3}{|c|}{$-79,005 a 966667$ Zona 06} \\
\hline & $120101000020 E 61000001 F 68849$ CF 0052641 C $40 C F 15632766 .$. & 540 & DEsIZAMIENTOS & MEDto & $-2,9001283333$ & \multicolumn{3}{|c|}{-79,00539666667 Zona 06} \\
\hline & $130101000020 E 6100000$ 1F68349CF005264IC 4DCF $15632766 .$. & 550 & DESLIZAMTENTOS & NEDTO & $-2,9001283333$ & \multicolumn{3}{|c|}{$-79,0058966667$ Zona 06} \\
\hline & $140101000020 E 61000001$ F $68349 C=0052641 C 40 \mathrm{CF} 15632766 .$. & 560 & DESLIZAMIENTOS & MEDIO & $-2,9001283333$ & \multicolumn{3}{|c|}{$\cdot 79,0058966667$ Zona 05.} \\
\hline 1 & $m$ & & 要 & & & \multicolumn{3}{|c|}{, } \\
\hline & II 9 & \multicolumn{6}{|c|}{$441 / 21$ 它WD } & \\
\hline
\end{tabular}

Se publican automáticamente las capas que se encuentran en la base de datos espacial, en el servidor de mapas GeoServer.

Se organiza y clasifica la información reportada, como se puede ver en la Figura 7 (Nombre del tipo de evento/Cantón/Año/Nombre de la capa). El nombre de la capa se asignará dependiendo del tipo de evento (código del evento, actualmente se está trabajando con deslizamientos), cantón, año y mes, por ejemplo: DLZMTS_CUENCA_201801.

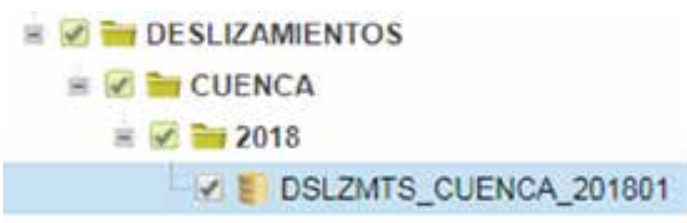

Figura 7. Organización y clasificación de la información reportada

Se visualiza la información en el visor de mapas de la IDE-UCuenca, cuya dirección de enlace es: http://ide.ucuenca.edu.ec/ide/alerts_tdt/

\section{PRUEBAS DE LA APLICACIÓN}

La aplicación ha sido probada en tres zonas de la ciudad de Cuenca que se han definido como áreas de estudio dentro del proyecto, Zona 1: Trigales Altos, Zona 2: Reina del Cisne (Acero et al. 2017) y Zona 3: Gapal-Universidad del Azuay (UDA). Para el levantamiento de información utilizando la aplicación móvil, se capacitó a 15 estudiantes de la asignatura de Geoinformática, que cursaban el octavo ciclo de la carrera de Informática de la Universidad de Cuenca, quienes levantaron un total de 493 puntos, de los cuales 196 corresponden a infraestructura y 297 a terreno. Los puntos están distribuidos de la 
siguiente manera: zona 1: 193 puntos, zona 2: 100 puntos, zona 3: 200 puntos. En la Figura 8 se puede observar una fotografía de una grieta en el sector Gapal-UDA, tomada a través de la aplicación móvil.

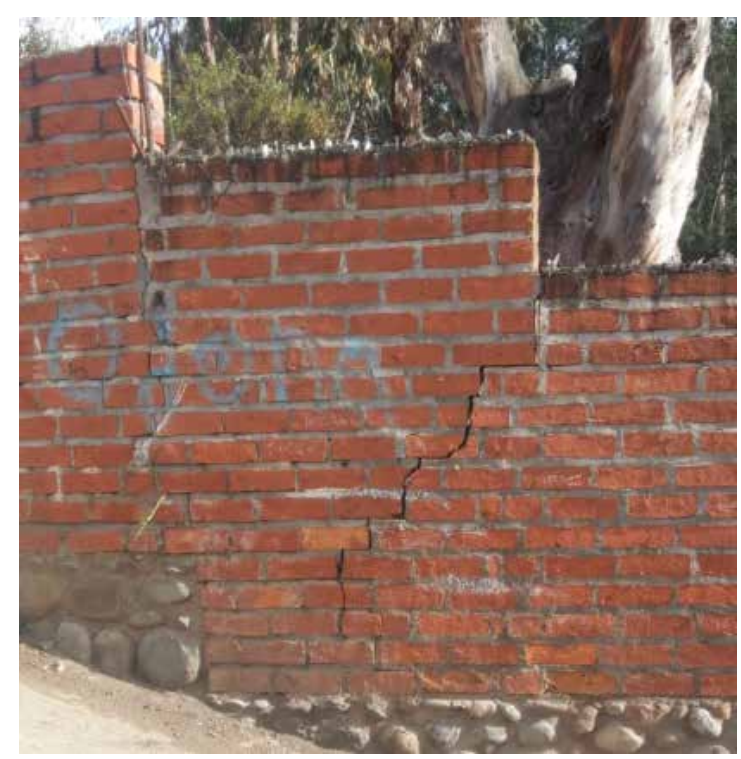

Figura 8. Fotografía sector Gapal - UDA

En la Figura 9, Figura 10 y Figura 11, se puede observar el total de puntos levantados en los 3 sectores, dentro del visor de la plataforma IDE-UCuenca.

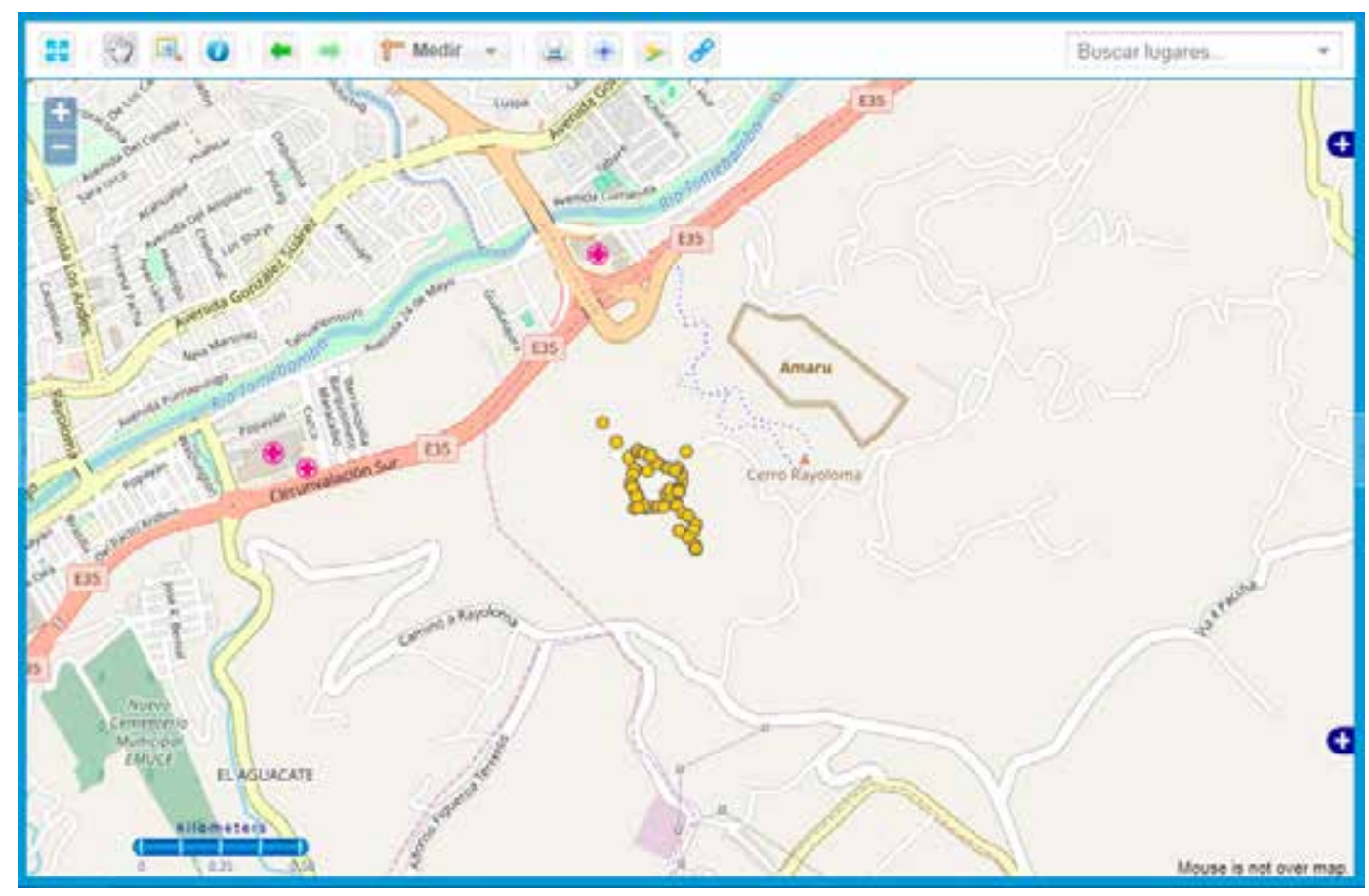

Figura 9. Puntos levantados por los usuarios en el sector Los Trigales 


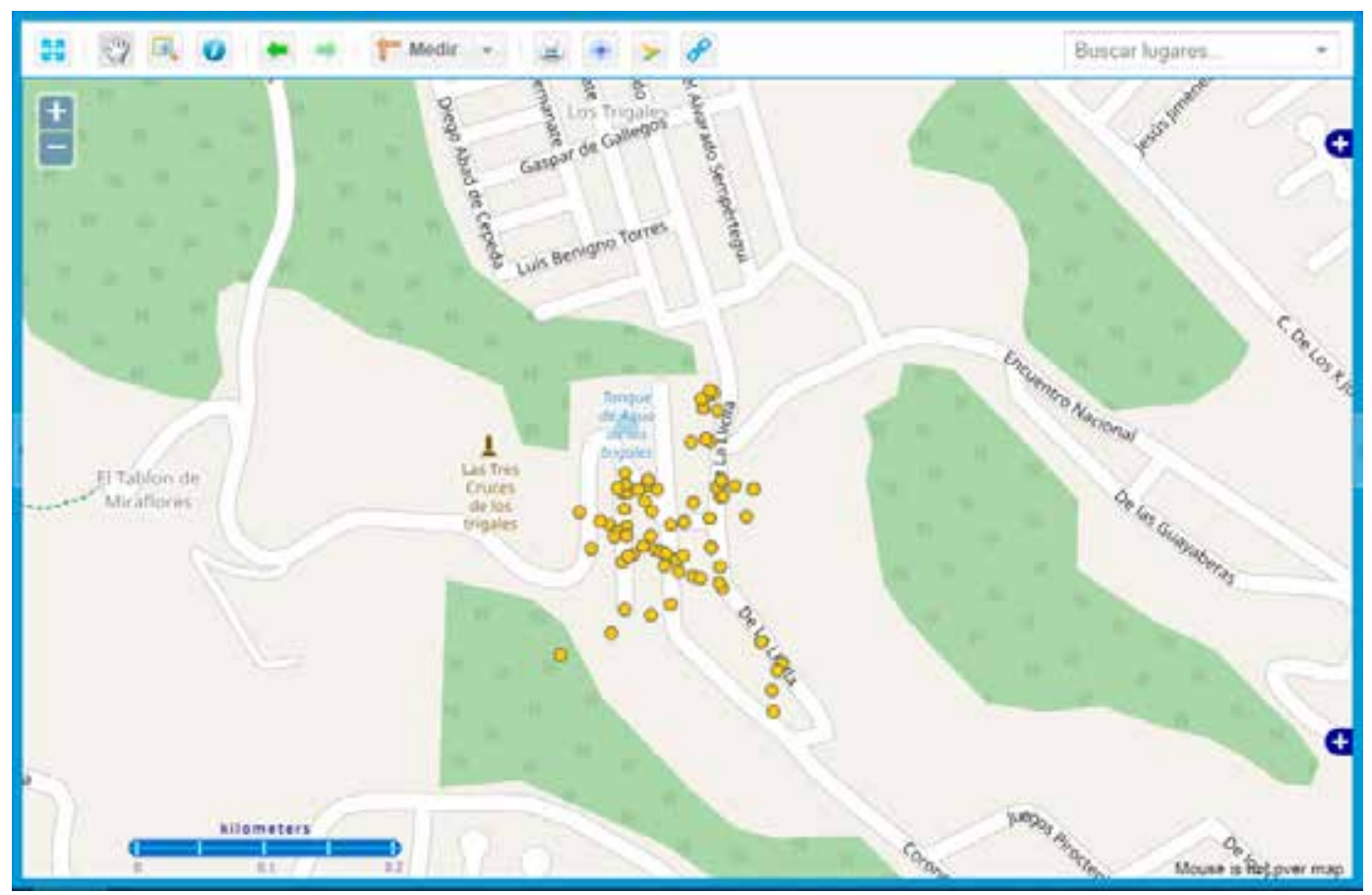

Figura 10. Puntos levantados por los usuarios en el sector Reina del Cisne

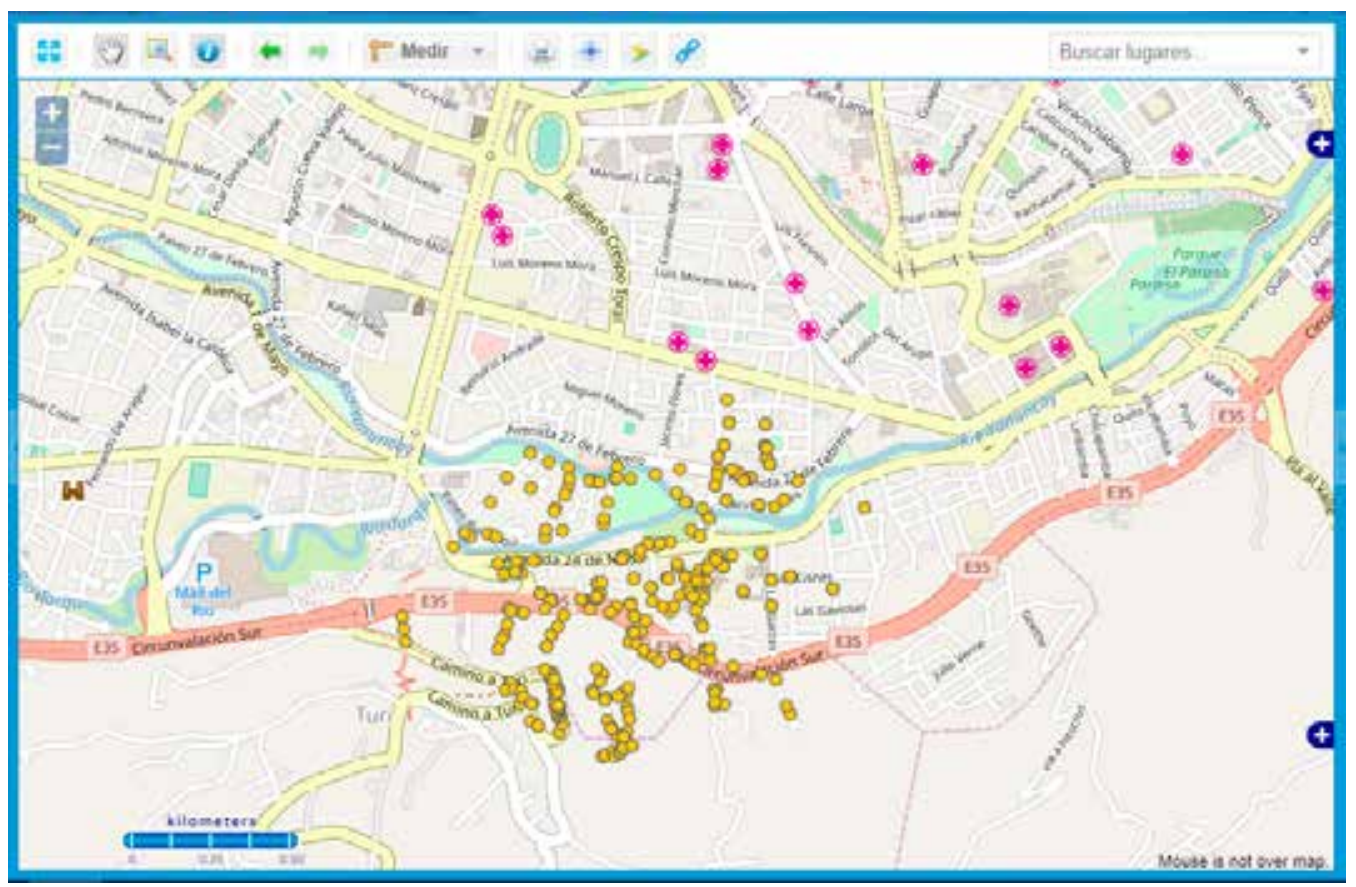

Figura 11. Puntos levantados por los usuarios en el sector Gapal-UDA 


\section{VALIDACIÓN DEL DISEÑO DE LA ENCUESTA}

Una vez implementada la aplicación móvil, se procedió a la validación de las preguntas de la encuesta por parte del personal de la SGR, que emitió observaciones y recomendaciones principalmente para añadir información de utilidad para la institución relacionada al uso del suelo, vulnerabilidad, resiliencia y detalles del deslizamiento. A continuación se presentan los esquemas correspondientes a los perfiles de ciudadano y técnico, con las modificaciones incluidas. Para una mayor comprensión los esquemas se han dividido en dos secciones, la primera contiene información general sobre el lugar y la segunda parte tiene información relacionada al deslizamiento.

En la Figura 12 se muestra la primera sección del perfil de ciudadano común.

En la Figura 13 se presenta un esquema con la información que el usuario deberá ingresar respecto al deslizamiento que desea reportar.

En la Figura 14 se muestra el esquema de la información general correspondiente al perfil técnico.

En la Figura 15 se presenta un diagrama con la información que el técnico deberá ingresar respecto al deslizamiento que desea reportar.

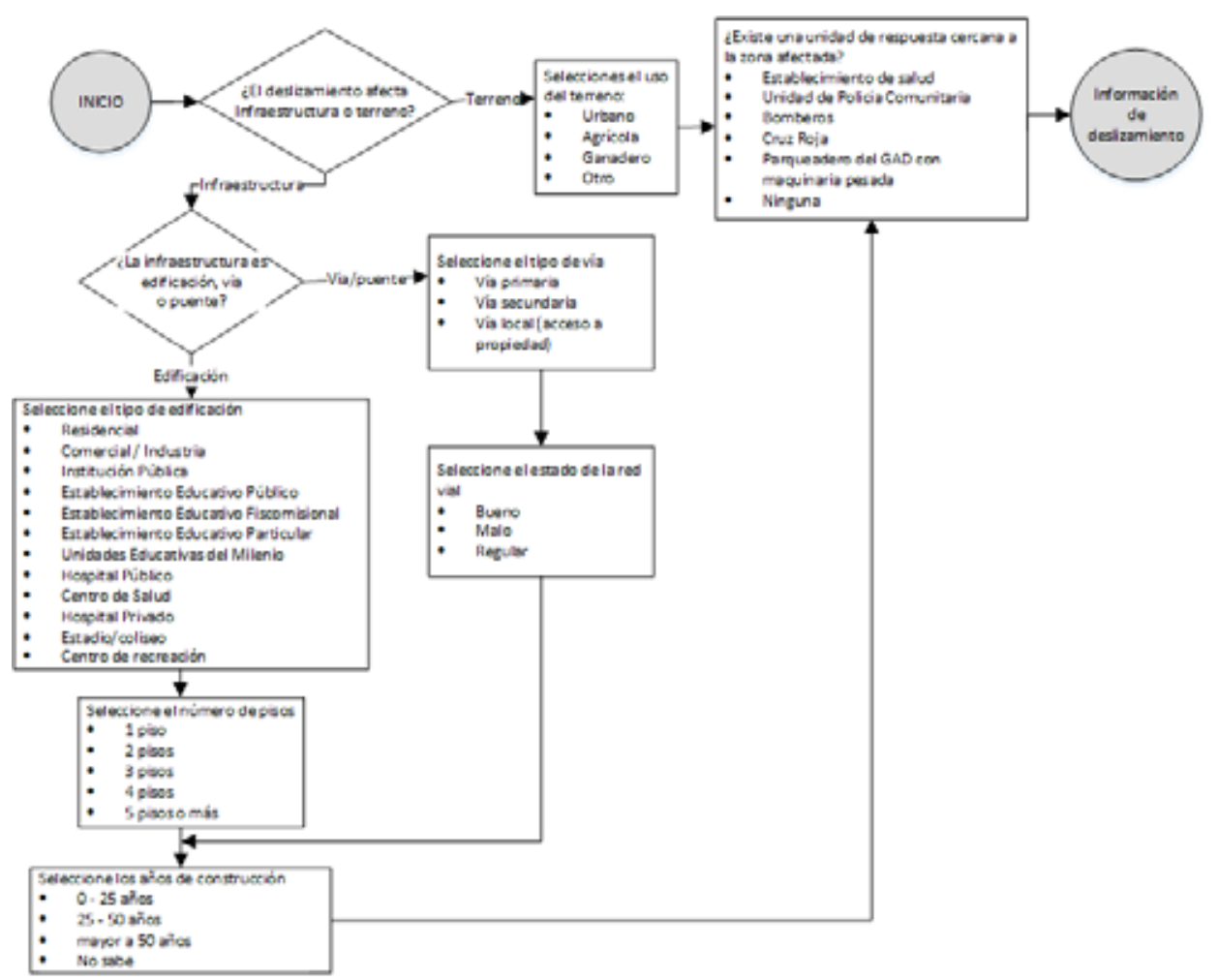

Figura 12. Encuesta al Ciudadano. Datos generales. 


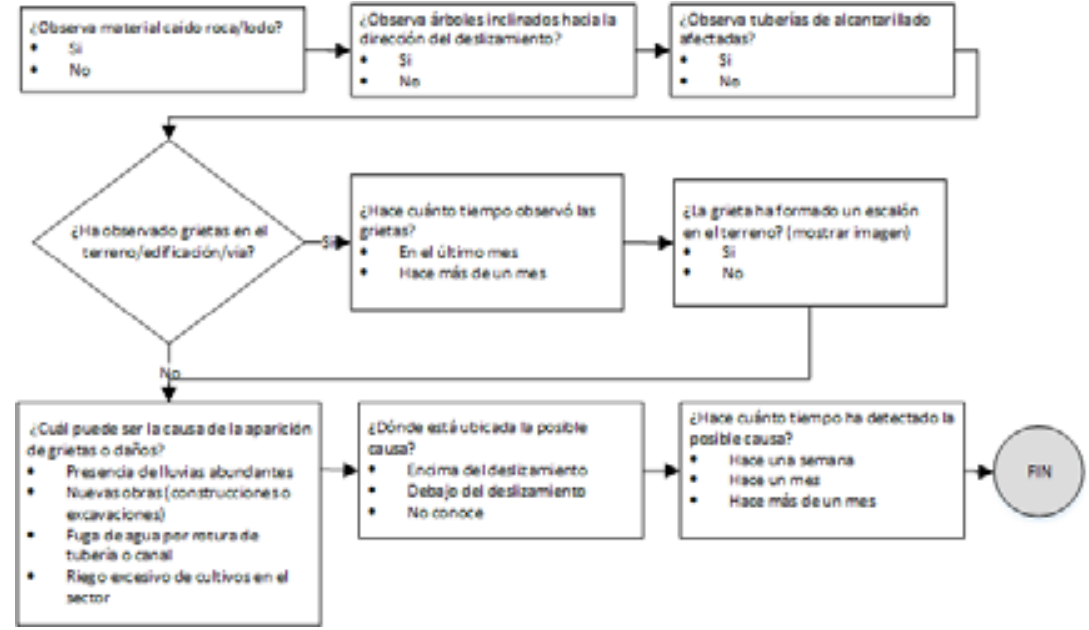

Figura 13. Encuesta al Ciudadano. Datos del deslizamiento

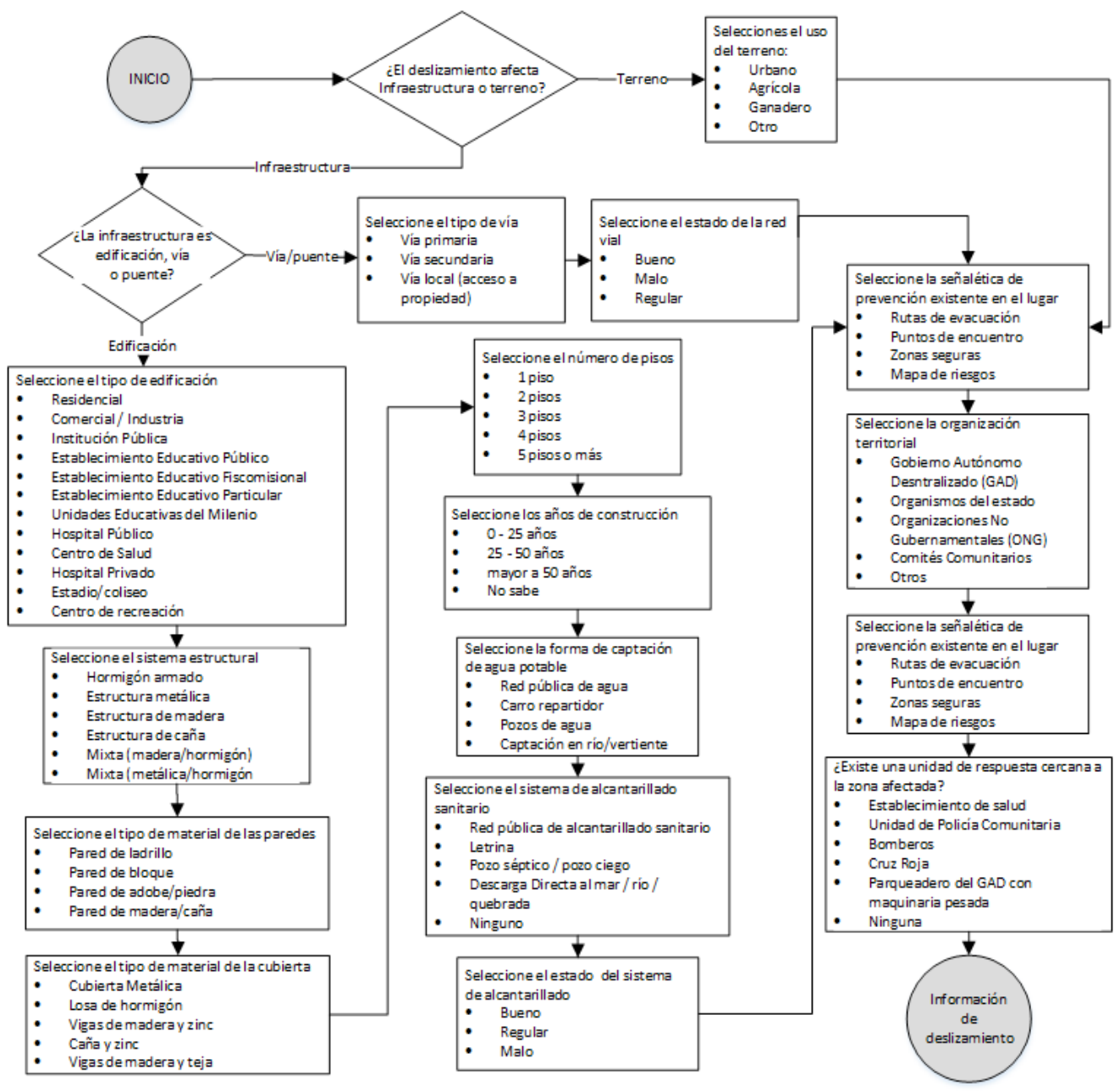

Figura 14. Encuesta al Personal Técnico. Datos generales. 


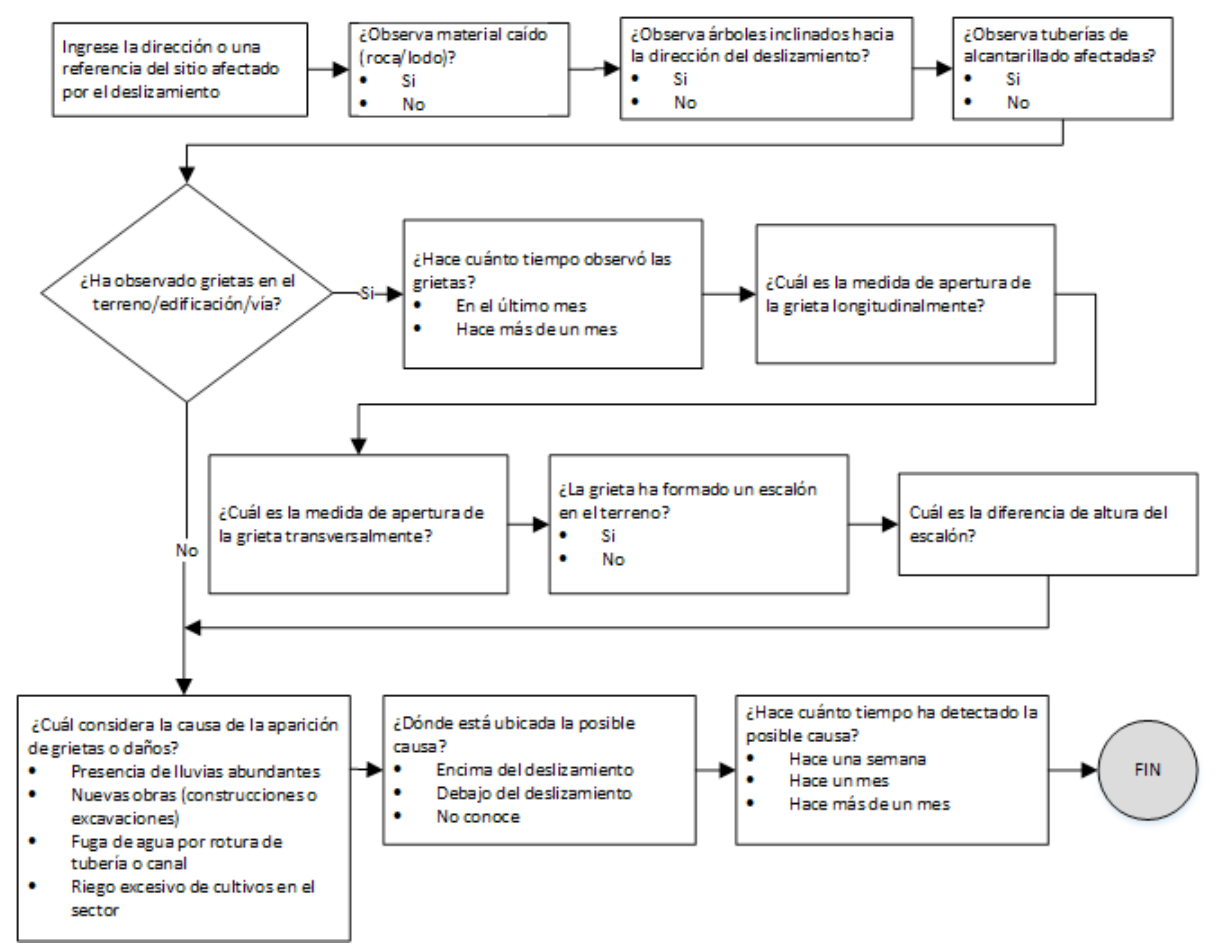

Figura 15. Encuesta al Personal Técnico. Datos del deslizamiento.

\section{CONCLUSIONES}

Según los datos analizados, en Ecuador no se han generado sistemas aproximados a los PP GIS por lo que este trabajo se presenta como una oportunidad para la promoción y fortalecimiento de estos sistemas. Como prueba piloto se realizó el levantamiento de información con estudiantes, simulando el uso de un ciudadano común, fue una primera aproximación importante que servirá de base para la interacción con la población.

El resultado de esta aplicación no sólo consiguió que la generación de información por los diferentes medios aporte a la planificación, sino incluso promovió el uso de dicha información por parte de otras organizaciones como es la SGR. Normalmente dicha entidad también genera información utilizando cierto tipo de encuestas, consideradas dentro del "Plan de respuesta ante desastres" (Ocles 2018), las que en este proyecto se integraron como parte de la aplicación móvil, que permite la participación ciudadana. Así, este aporte es acoplado a una estructura validada por la SGR lo que permite que el aporte del ciudadano, en caso de eventos que puedan afectar a vidas o infraestructura, también sean parte de una alerta temprana. Dicha información puede ser capturada por el ciudadano a través de la aplicación móvil y enviada en tiempo real para que la SGR pueda analizarla y activar una alerta temprana usando la plataforma IDE-UCuenca. Si bien es cierto, esta activación dependerá de muchos otros factores, sin embargo, la posibilidad de tener mayor información relevante y en tiempo real, contribuye a un mejor análisis y tiempo de respuesta de la entidad. 


\section{AGRADECIMIENTOS}

Este trabajo ha sido financiado por la Dirección de Investigación de la Universidad de Cuenca (DIUC) bajo el XV concurso de proyectos, con el nombre "Geoprocesamiento IDE como apoyo al análisis de vulnerabilidades y planificación territorial" 2017-2018. Especial agradecimiento al Ing. Ricardo Peñaherrera e Ing. Leonardo Espinosa de la SGR y a la Fundación RedCEDIA por su apoyo al desarrollo de las IDEs académicas por medio del GT-IDE.

\section{REFERENCIAS BIBLIOGRÁFICAS}

Abhik, Dhekar, y Durga Toshniwal. 2013. «Sub-event detection during natural hazards using features of social media data». En 22nd International Conference on World Wide Web, 783-88. New York, NY, USA. https://doi.org/10.1145/2487788.2488046.

Acero, P, M Caldas, P Mejía, C Pesántez, R Piedra, C Morocho, y E Acosta. 2017. «Tipología y cinética del deslizamiento Reina del Cisne (Cuenca) a partir de fotografías aéreas, GPS, escáner láser terrestre y ensayos geotécnicos del suelo.» 14 (2): 50-56.

Callahan, Kathe. 2007. «Citizen Participation: Models and Methods». International Journal of Public Administration 30 (11): 1179-96. https://doi.org/10.1080/01900690701225366.

Castells, Manuel. 2008. «Comunicación, poder y contrapoder en la sociedad red (II). Los nuevos espacios de la comunicación». Telos 75: 11-23.

Morocho, Villie. 2013. «Aportes a la lectura de la vulnerabilidad, a partir de los resultados de los análisis realizados a nivel cantonal». http://repositorio.cedia.org.ec/handle/123456789/866.

Morocho, Villie, y Fabian Santander. 2015. «De los rígidos códigos internos de una IDE a un ambiente gráfico de administración y gestión: IDE Ucuenca V3.5». Revista GEOESPACIAL, 2015.

Ocles, Alexandra. 2018. «Plan Nacional de Respuesta SGR». https://www.gestionderiesgos. gob.ec/wp-content/uploads/downloads/2018/08/Plan-Nacional-de-Respuesta-SGRRespondeEC.pdf.

Pacurucu, Natalia, y Villie Morocho. 2017. «Los PP GIS como geo-herramienta para el mapeo de zonas vulnerables, en el contexto de la planificación territorial: una revisión bibliográfica» 14 (2): 116-25.

Pearce, Laurie. 2003. «Disaster Management and Community Planning, and Public Participation: How to Achieve Sustainable Hazard Mitigation». Natural Hazards, 211-28.

Sheppard, Eric, Helen Couclelis, Stephen Graham, James W Harrington, y Harlan Onsrud. 1999. "Geographies of the information society». International journal of geographical information science 13 (8): 797-823.

Sieber, Renee. 2006. «Public Participation Geographic Information Systems: A Literature Review and Framework». Annals of the Association of American Geographers 96 (3): 491-507. https://doi.org/10.1111/j.1467-8306.2006.00702.x. 